\title{
IMPROVEMENT OF SCALE-XSPROC MULTIGROUP CROSS SECTION PROCESSING BASED ON THE CENTRM POINTWISE SLOWING DOWN CALCULATION
}

\author{
Kang Seog Kim ${ }^{1}$, Andrew M. Holcomb ${ }^{1}$, Friederike Bostelmann ${ }^{1}$, Dorothea Wiarda ${ }^{1}$, and \\ William Wieselquist ${ }^{1}$ \\ ${ }^{1}$ Oak Ridge National Laboratory \\ 1 Bethel Valley Road, Oak Ridge, TN 37831, USA
}

kimk1@ornl.gov, holcombam@ornl.gov, bostelmannf@ornl.gov,wiardada@ornl.gov, wieselquiswa@ornl.gov

\begin{abstract}
The SCALE-XSProc multigroup (MG) cross section processing procedure based on the CENTRM pointwise slowing down calculation is the primary procedure to process problemdependent self-shielded MG cross sections and scattering matrices for neutron transport calculations. This procedure supports various cell-based geometries including slab, 1-D cylindrical, 1-D spherical and 2-D rectangular configurations and doubly heterogeneous particulate fuels. Recently, this procedure has been significantly improved to be applied to any advanced reactor analysis covering thermal and fast reactor systems, and to be comparable to continuous energy (CE) Monte Carlo calculations. Some reactivity bias and reaction rate differences have been observed compared with CE Monte Carlo calculations, and several areas for improvement have been identified in the SCALE-XSProc MG cross section processing: (1) resonance self-shielding calculations within the unresolved resonance range, (2) $10 \mathrm{eV}$ thermal cut-off energy for the free gas model, (3) on-the-fly adjustments to the thermal scattering matrix, (4) normalization of the pointwise neutron flux, and (5) fine MG energy structure. This procedure ensures very accurate MG cross section processing for high-fidelity deterministic reactor physics analysis for various advanced reactor systems.
\end{abstract}

KEYWORDS: SCALE-XSProc, multigroup cross section processing, slowing down calculation

\section{INTRODUCTION}

The SCALE-XSProc [1] multigroup (MG) cross section processing procedure includes two methods to process problem-dependent self-shielded MG cross sections [2] for transport calculations: (1) a Bondarenko method [3], and (2) a pointwise (PW) slowing down calculation. First, the fast-executing Bondarenko

\footnotetext{
Notice: This manuscript has been authored by UT-Battelle, LLC, under contract DE-AC05-00OR22725 with the US Department of Energy (DOE). The US government retains and the publisher, by accepting the article for publication, acknowledges that the US government retains a nonexclusive, paid-up, irrevocable, worldwide license to publish or reproduce the published form of this manuscript, or allow others to do so, for US government purposes. DOE will provide public access to these results of federally sponsored research in accordance with the DOE Public Access Plan (http://energy.gov/downloads/doe-public-access-plan).
} 
method is applied to all energy groups for various reaction cross sections of all materials using the BONAMI [1] method kernel within XSProc. In this method, MG data at problem-specific conditions are interpolated from the tabulated self-shielding factors in the MG library. The second approach is to generate problem-dependent MG data directly from PW data within the energy range in which the Bondarenko method is not desirable, which is typically the resolved resonance and thermal ranges. Generally, the PW energy range should encompass the resolved resonance ranges of all nuclides that impact the spectral fine structure; however, MG data in very high and low energy ranges are always self-shielded with the Bondarenko method. The combined MG and PW calculation is performed by the CENTRM $[1,4]$ method kernel within XSProc, which computes neutron spectra on a fine energy mesh within the PW energy range using various approximations to the Boltzmann equation. In the energy region outside the specified PW range, CENTRM performs an MG calculation so that a full energy range solution is obtained. Finally, a submodule of XSProc called PMC computes problem-specific MG data for groups within the PW energy range of the CENTRM calculation and replaces the more approximate values from BONAMI. PMC uses the CENTRM spectra for each spatial region as a problem-dependent weight function for averaging energydependent data in the PW libraries into MG data. The resulting problem-specific cross section library is passed to higher-dimensional MG transport calculations performed with other SCALE modules.

For non-light water reactor (non-LWR) analysis, reactivity bias and reaction rate differences were observed compared with continuous energy (CE) Monte Carlo calculations. Several areas for improvement were identified in the SCALE-XSProc MG cross section processing: (1) resonance self-shielding calculations within the unresolved resonance range (URR) [5], (2) the $5 \mathrm{eV}$ thermal cut-off energy for the free gas model, (3) on-the fly adjustments to the thermal scattering matrix, (4) normalization of the PW neutron flux, and (5) multigroup structure [5,6]. Since the PW neutron flux moments are used as weighting functions to obtain MG data, there are limits to the need for increased accuracy based on how this approach affects quantities of interest in criticality safety and reactor physics applications. This work has reduced a significant amount of the difference compared with CE Monte Carlo by developing a new analytic probability table method for the URR, increasing the cut-off energy to $10 \mathrm{eV}$, incorporating a new procedure to explicitly reconstruct thermal scattering matrices, increasing robustness of the flux normalization, and improving the energy group structure for any advanced reactor analysis.

\section{IMPROVEMENT OF SCALE-XSPROC}

\subsection{SCALE-XSProc Cross Section Processing Procedure}

A flowchart of the SCALE modules executed for self-shielding is shown in Figure 1. First, the fastexecuting Bondarenko method is applied to all energy groups for the total, elastic, capture, and fission cross sections of all materials by using BONAMI. In this method, MG data at problem-specific conditions are interpolated from the tabulated self-shielding factors on the MG library. While the analytical flux expression used to process Bondarenko shielding factors is reasonable for higher energy groups, it tends to break down at lower energies where the narrow resonance (NR) approximation is not accurate.

SCALE 6 provides other approaches to generate problem-dependent MG data directly from PW data within the energy range in which the Bondarenko method is not desirable; typically, this includes the resolved resonance and thermal ranges for criticality safety and reactor physics applications. The energy range of interest is divided into the upper multigroup (UMR), PW, and lower multigroup (LMR) ranges, as defined by the input energy boundaries $E_{\max }$ and $E_{\min }$. These have default values of $20 \mathrm{keV}$ for $E_{\max }$ and $10^{-3} \mathrm{eV}$ for $E_{\min }$ in SCALE 6. Generally, it is recommended that the PW energy range should encompass the resolved resonance ranges of all nuclides that impact the spectral fine-structure; however, MG data in the UMR and LMR are always self-shielded with the Bondarenko method. Thus, it is not necessary to perform a CE calculation within the energy range where the Bondarenko approximation is adequate. This calculation is performed by the CENTRM code, which computes CE neutron spectra within the PW energy range using 
various approximations to the Boltzmann equation, including an infinite medium model, a two-region (2R) method for lattices, and one- or two-dimensional transport calculation with either the collision probability or discrete ordinates method. In the energy region outside of the specified PW range, CENTRM performs an MG calculation so that a full energy range solution is obtained.

The PMC module computes problem-specific MG data for groups within the PW energy range of the CENTRM calculation and replaces the more approximate values from BONAMI. PMC uses the CENTRM spectra for each spatial region as a problem-dependent weight function for averaging energy-dependent data in the PW libraries into MG data. The resulting application-specific cross section library is passed to higher dimensional MG transport calculations performed with other SCALE modules.

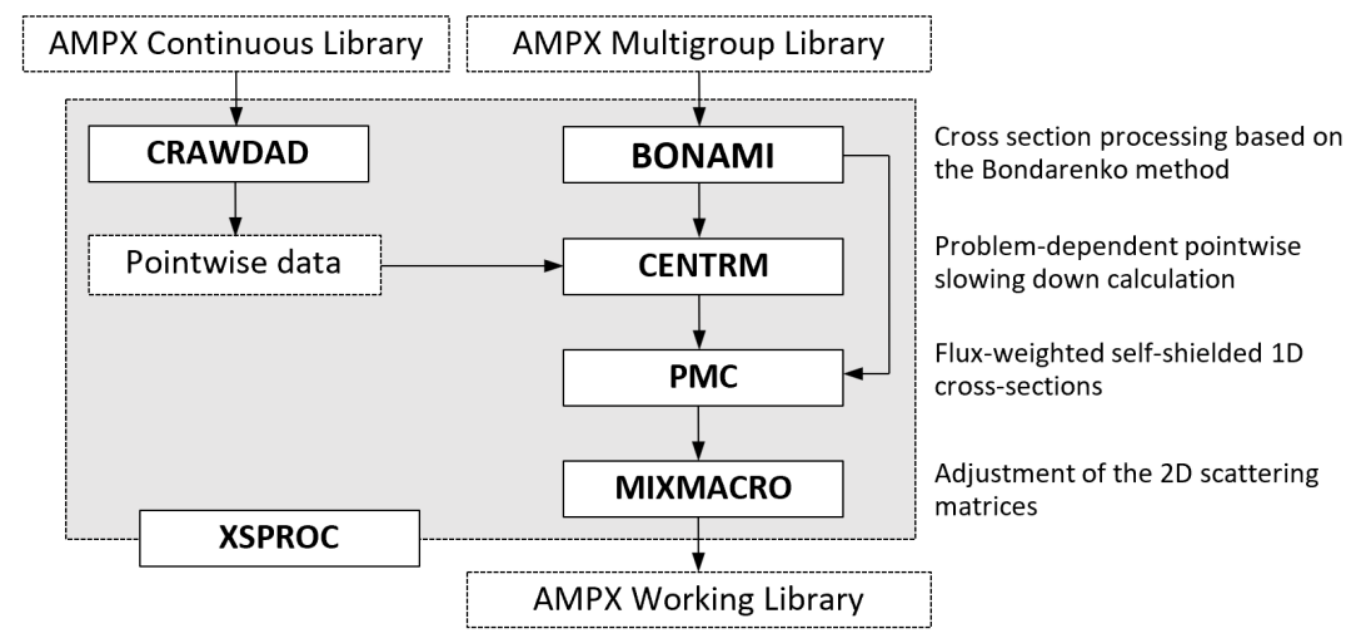

Figure 1. Flowchart of modules to generate problem-dependent multigroup data.

\subsection{Unresolved Resonance Self-Shielding}

The effective self-shielded cross section of reaction $x$ of a nuclide $i$ for an URR energy group $g$ can be obtained from the following weighting equation:

$$
\sigma_{x, g, i}=\frac{\int_{g} \sum_{m} p_{i}^{m} \sigma_{x, i}^{m}(E) \phi^{m}(E) d E}{\int_{g} \sum_{m} p_{i}^{m} \phi^{m}(E) d E},
$$

where

$$
\begin{aligned}
& \sigma_{x}^{m} \quad=\text { a cross section level } m \text { of reaction } x \text { in the URR probability table, } \\
& p^{m} \quad=\text { a probability of the level } m \\
& \sigma_{x, g} \quad=\text { a self-shielded cross section of reaction } x, \text { and } \\
& \phi^{m}(E)=\text { scalar flux at the level } m .
\end{aligned}
$$

The cross section levels $\left(\sigma_{x}^{m}\right)$ and probabilities $\left(p^{m}\right)$ are given at the specified energy points and can be expanded to any URR energy point. If the MG energy boundaries are very narrow, the level-wise scalar fluxes can be obtained from the fixed source MG transport calculation, which can be used in Eq. (1) to estimate the MG self-shielded cross sections. However, this calculation will be highly time consuming. The NR approximation can be a good solution for the URR energy range in which the PW neutron spectrum can be approximated in terms of total and background cross sections, as follows: 


$$
\phi^{m}(E)=\frac{W(E)}{N_{i} \sigma_{t, i}^{m}(E)+\sum_{j \neq i} N_{j} \sigma_{t, j}(E)+\Sigma_{e}(E)},
$$

where $\Sigma_{e}$ is a macroscopic equivalence cross section, $N_{i}$ is an atomic number density of a target nuclide $i$, and $W(E)$ is a PW weighting function. The equivalence cross section can be obtained from the Dancoff factor using two different approaches. The first approach is to estimate MG URR self-shielded cross sections on the fly for a given geometry and material composition by directly using probability tables. When the energy group width is very narrow, Eq. (1) can be rewritten with Eq. (2) as follows:

$$
\begin{gathered}
\sigma_{x, g, i}=\frac{\sum_{m} \frac{p_{i}^{m} \sigma_{x, i, g}^{m}}{\sigma_{t, i, g}^{m}+\sigma_{0, i, g}}}{\sum_{m} \frac{p_{i}^{m}}{\sigma_{t, i, g}^{m}+\sigma_{0, i, g}}} \\
\sigma_{0, i, g}=\frac{1}{N_{i}}\left(\sum_{j \neq i} N_{j} \sigma_{t, j, g}+\Sigma_{e, g}\right) .
\end{gathered}
$$

A Bondarenko iteration is required to obtain converged self-shielded cross sections by considering resonance interference when multiple resonance nuclides exist. The second approach consists of preparing the self-shielded cross section table as a function of background cross section $\left(\sigma_{0}\right)$ in advance, and then estimating the background cross sections on the fly so that the self-shielded cross sections can be read from the precalculated resonance table. This approach also requires the Bondarenko iteration to consider resonance interference. The SCALE/AMPX [7] procedure uses the second approach, and the AMPX MG library includes the URR self-shielded cross section tables. The SCALE-BONAMI estimates the effective self-shielded cross sections for a given geometry and composition.

\subsection{Pointwise Neutron Flux Normalization}

Abnormal PW neutron flux normalization has been observed in CENTRM as shown in Figure 2. Since the CENTRM PW flux moments are used as weighting functions to obtain self-shielded cross sections and scattering matrices, there is no significant impact on transport calculations. However, this issue prohibits the introduction of any equivalence parameters, such as super-homogenization factors, to conserve reaction rates for the following transport calculations. CENTRM includes a lumping procedure to efficiently estimate nuclide-wise scattering source based on atomic mass, which would not work correctly and would result in unusual PW neutron spectra. Now no lumping procedure is engaged in calculating scattering source as a default. The lumping capability will be improved to enhance computational efficiency without introducing any abnormal flux normalization.

\subsection{Thermal Cutoff Energy for Free Gas Model}

The SCALE MG procedure based on the CENTRM PW slowing down calculation has typically been used the $5.0 \mathrm{eV}$ thermal cutoff energy for free gas model for thermal scattering. The CE Monte Carlo codes such as KENO and MCNP use much higher cutoff energies for the free gas model to be user specific and to have $400 \mathrm{KT}$, respectively, where $K$ is the Boltzmann constant and $T$ is the temperature in Kelvin. Since ENDF/BVII.1 provides bounding nuclide thermal scattering data called $S(\alpha, \beta)$ up to $5.0 \mathrm{eV}$, the free gas model is used for $5.0 \mathrm{eV}$ through the thermal cutoff energy for bound nuclides and zero to the thermal cutoff energy for other non-bound nuclides. The $5.0 \mathrm{eV}$ thermal cutoff energy for the free gas model in CENTRM was 
relatively successful for light water reactor analysis. However, the $5.0 \mathrm{eV}$ cutoff would result in significant reaction rate differences for graphite-moderated systems. Figure 3 provides reaction rate analysis with 5.0 $\mathrm{eV}$ and $10.0 \mathrm{eV}$ cutoff energies between CE-KENO- and CENTRM-based MG-KENO for the single heterogeneous gas-cooled reactor fuel. Reaction differences of the circle B in Figure 3 (a) are significantly improved by using the $10.0 \mathrm{eV}$ cutoff as shown in Figure 3 (b). Reaction rate differences of the circle A in Figure 3 came from poor thermal scattering matrices and are discussed in Section 2.5.

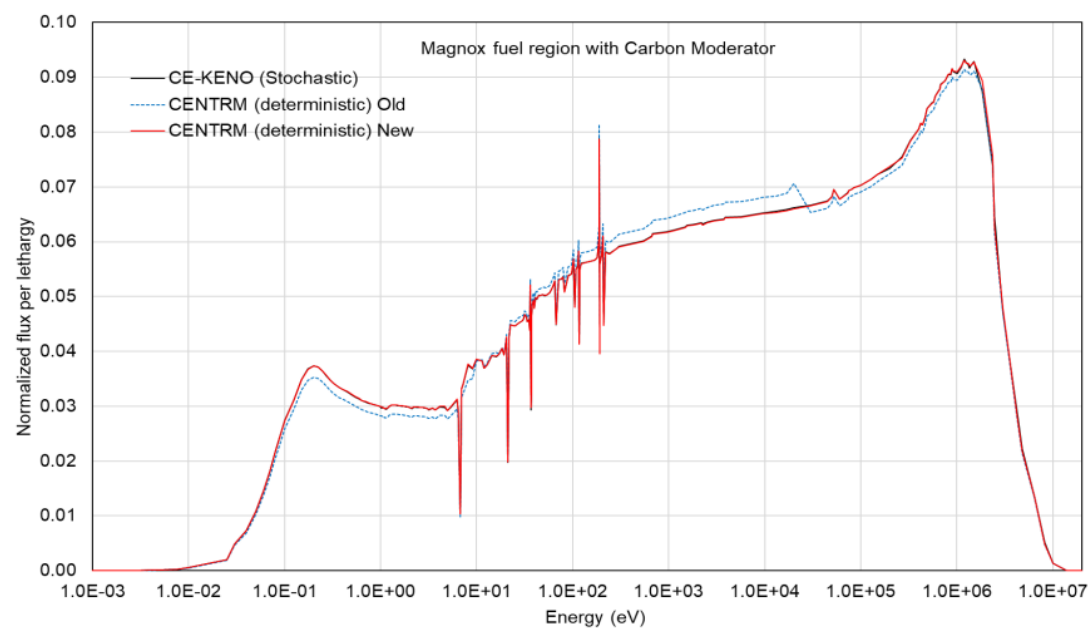

Figure 2. Neutron flux normalization in CENTRM.

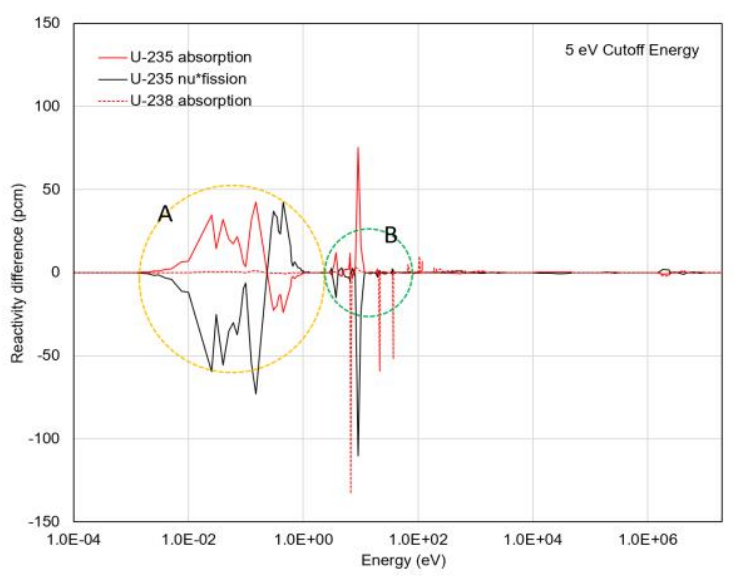

(a) $5.0 \mathrm{eV}$ cutoff

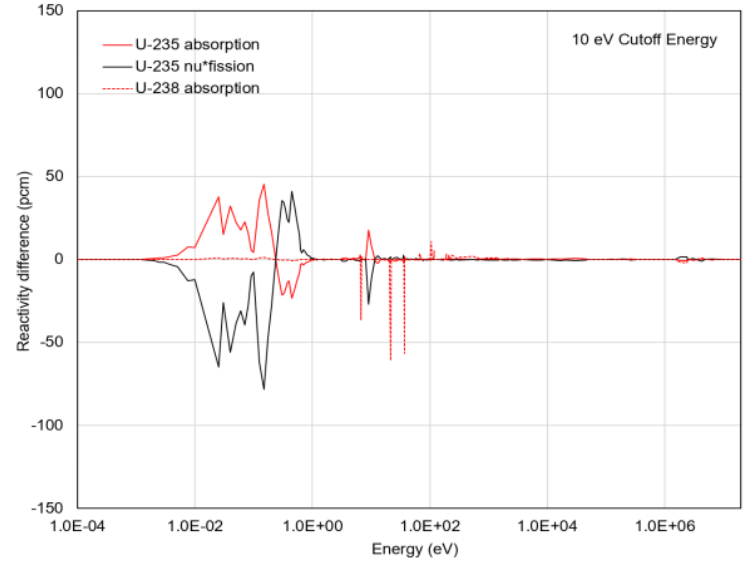

(b) 10.0 eV cutoff

Figure 3. Comparison of reaction rate analysis between 5.0 and $10.0 \mathrm{eV}$ cutoff energies.

\subsection{On-the-fly Thermal Scattering Matrices}

The circle A in Figure 3 (a) indicates reaction rate differences between the CENTRM based SCALE MG and CE Monte Carlo results which come from poor thermal spectra due to poor MG scattering matrices. Figure 4 compares neutron spectra between CENTRM and MCNP [8] for the Magnox fuel [9] with the graphite moderator at $1,200 \mathrm{~K}$. A significant difference in neutron spectra was observed at the thermal peak 
at around $0.15 \mathrm{eV}$. The difference was the result of the poor interpolation with PW-bound nuclide $\mathrm{S}(\alpha, \beta)$ scattering kernel data. The existing peak-based interpolation scheme was improved, and a new unit-based interpolation scheme was implemented into CENTRM for the $S(\alpha, \beta)$ data treatment. The CENTRM PW neutron spectra with both interpolation schemes were significantly improved and are very consistent with the MCNP spectra, as shown in Figure 4.

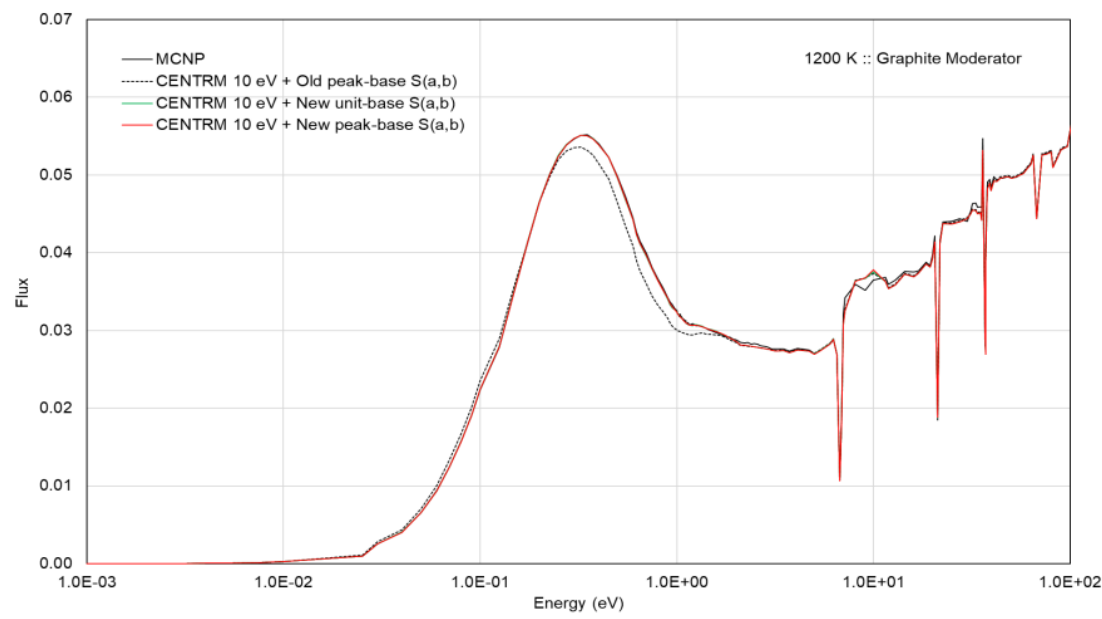

Figure 4. Comparison of CENTRM and MCNP thermal neutron spectra.

Even though very precise PW neutron spectra were available from the PW slowing down calculation, scattering matrices were not explicitly updated but were adjusted only for level-based self-shielded scattering cross sections for energies below the thermal cutoff. As shown in Figure 5, a significant difference was observed between the CENTRM and XSDRN neutron spectra for the homogeneous mixture problem, including graphite, which was selected to avoid having angle-dependent total cross sections and to exclude high-order terms in scattering matrices. The XSDRN code is a SCALE MG deterministic transport solver. A new capability was implemented to explicitly reconstruct MG thermal scattering matrices using PW scattering kernel data and flux moments. Figure 5 shows a perfect agreement between the CENTRM and XSDRN neutron spectra when using the explicit reconstruction of thermal scattering matrices in XSProc.

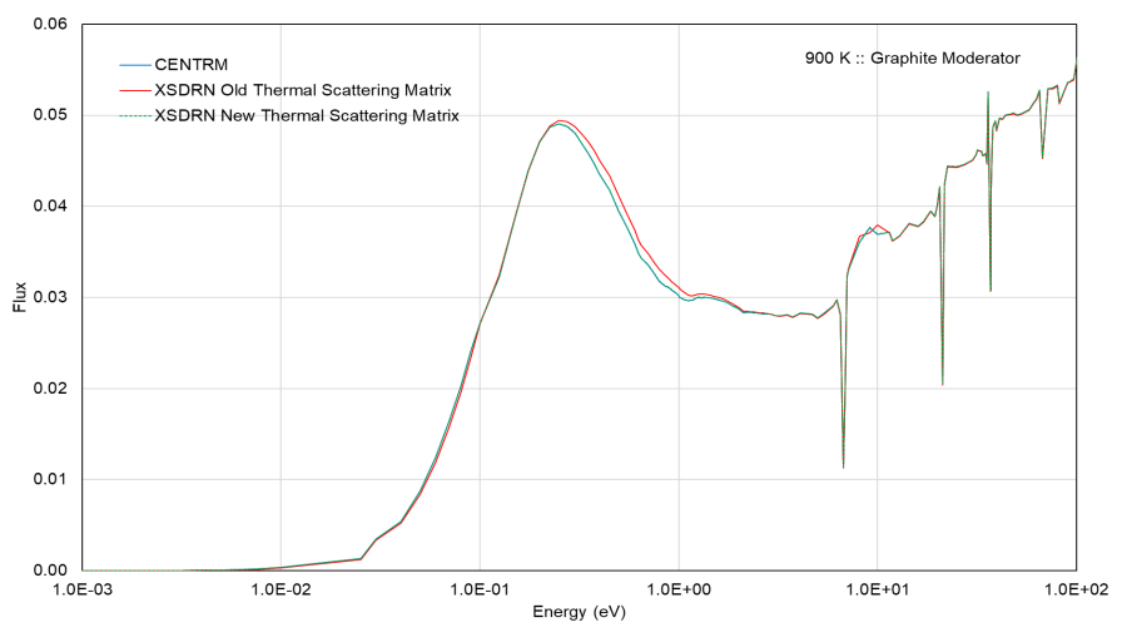

Figure 5. Comparison of thermal neutron spectra between CENTRM and XSDRN. 


\subsection{Ultra-Fine Group AMPX Library}

SCALE 6.2 provides the ENDF/B-VII.1 AMPX 56- and 252-group libraries for reactor physics analysis. These libraries introduce significant reactivity bias for fast reactor analysis. A new 1,597-group structure [6] was developed for any advanced reactor analysis including LWRs; prismatic block and pebble-bed hightemperature gas-cooled reactors; thermal and fast molten salt reactors; and various fast reactor systems. In addition, a 302-group structure was developed only for fast reactor analysis. The ENDF/B-VII.1 and VIII.0 AMPX 302- and 1,597-group libraries will be available for SCALE 6.3, which will be released in 2020.

The 1,597-group structure was developed based on the AMPX 252-group and $\mathrm{MC}^{2}-3$ ultra-fine group (UFG) structures. The energy range from $0.1 \mathrm{keV}$ to $20 \mathrm{MeV}$ is divided into 1,323 groups by referring the $\mathrm{MC}^{2}-3$ UFG structure to explicitly represent broad resonances of intermediate-weight nuclides. The energy range from $10^{-5} \mathrm{eV}$ to $0.1 \mathrm{keV}$ is represented by 274 groups based on the AMPX 252-group structure. An extensive benchmark suite was developed to verify the AMPX 1,597-group library and the SCALE-XSProc cross section processing procedure, which will also be available in SCALE 6.3. Table I summarizes the benchmark suite and the applicability of the AMPX MG libraries.

Table I. Applicability of the AMPX MG libraries to various reactor types.

\begin{tabular}{|l|c|c|c|c|}
\hline \multirow{2}{*}{ Reactor type } & \multirow{2}{*}{ Spectrum } & \multicolumn{3}{c|}{ AMPX library applicability } \\
\cline { 3 - 5 } & & 1,597-group & 252-group & 302-group \\
\hline Pressurized Water Reactor & Thermal & Yes & Yes & No \\
\hline Boiling Water Reactor & Thermal; fast & Yes & Yes/No ${ }^{[a]}$ & No \\
\hline Advanced Burner Test Reactor & Fast & Yes & No & Yes \\
\hline EBR-II & Fast & Yes & No & Yes \\
\hline OECD-NEA MET-1000 & Fast & Yes & No & Yes \\
\hline OECD-NEA MOX-3600 & Fast & Yes & No & Yes \\
\hline Thermal Molten Salt Reactor & Thermal & Yes & Yes & No \\
\hline Fast Molten Salt Reactor & Fast & Yes & No & Yes \\
\hline Pebble Bed Gas-Cooled Reactor & Thermal & Yes & Yes & No \\
\hline Prismatic Gas-Cooled Reactor & Thermal & Yes & Yes & No \\
\hline
\end{tabular}

${ }^{[a]}$ Large reactivity bias for very high void fraction $>99 \%$

\section{CONCLUSIONS}

The standard SCALE-XSProc procedure processes effective MG neutron cross sections and scattering matrices for use in MG neutron transport calculations, using the Bondarenko approach with the AMPX MG library and performing the PW slowing down calculations. This standard procedure has been successfully applied to LWR analysis in the past. However, various minor issues introduced reactivity bias, and the procedure was not widely applied for fast spectrum simulations, including very high-void boiling water reactor and fast reactor problems. Recent improvements discussed in this study have resolved multiple issues regarding thermal and fast reactor analyses. The results of the verification studies show that the SCALE-XSProc cross section processing procedure with the new AMPX MG libraries provides reliable accuracy for advanced thermal and fast reactor analyses.

Currently, the CENTRM capability is comparable to the CE Monte Carlo codes. However, there are still minor pending complications, such as fixed thermal cutoff energy, no epithermal upscattering, and limited 
geometrical capability. Future investigations will focus on resolving the pending issues and optimizing the energy group structure to reduce the number of energy groups for any reactor system, since the 1,597-group structure requires a significant computational burden. In addition, an internal energy group collapsing procedure will be developed to achieve advantageous computational efficiency without loss of accuracy for large-scale problems.

\section{ACKNOWLEDGMENTS}

This research was supported by the US Nuclear Regulatory Commission Office of Research.

\section{REFERENCES}

1. B. T. Rearden, M. A. Jessee, SCALE Code System, ORNL/TM-2005/39, Version 6.2, Oak Ridge National Laboratory, Oak Ridge, Tennessee. Available from the Radiation Safety Information Computational Center as CCC-834 (2016).

2. M. L. Williams, "Resonance Self-Shielding Methodologies in SCALE 6," Nucl. Technol., 174(2), pp. 149-168 (2011).

3. I. I. Bondarenko, Ed., Group Constants for Nuclear Reactor Calculations, Consultants Bureau, New York City, USA (1964).

4. M. L. Williams, M. Asgari, "Computation of Continuous-Energy Neutron Spectra with Discrete Ordinates Transport Theory," Nucl. Sci. Eng., 121(2), 173-201 (1995).

5. K. S. Kim, M. L. Williams, A. Holcomb, D. Wiarda, B. K. Jeon, W. S. Yang, "The SCALE/AMPX Multigroup Cross Section Processing for Fast Reactor Analysis," Ann. Nucl. Energ., 132, 161-171 (2019).

6. K. S. Kim, F. Bostelmann, A. M. Holcomb, G. Ilas, and W. A. Wieselquist, "Verification of the ENDF/B-VII.1 and VIII.0 AMPX 1597-Group Libraries for Advanced Reactor Analysis," Proceeding of the Int. Conf. on Mathematics and Computational Methods applied to Nuclear Science and Engineering (M\&C 2019), Portland, OR, USA, August 25-29, 2019 pp. 2836-2845 (2019).

7. D. Wiarda et al., "AMPX-6: A Modular Code System for Processing ENDF/B," ORNL/TM-2016/43, Oak Ridge National Laboratory. Available from the Radiation Safety Information Computational Center as CCC-834 (2016).

8. X-5 Monte Carlo Team, "MCNP - A General Monte Carlo N-Particle Transport Code Version 5," LAUR-03-1987 (2008).

9. S.E. Jensen, E. Nonbol, "Description of the Magnox Type of Gas Cooled Reactor (MAGNOX)," NKS2 ISBN 87-7893-050-2, Riso National Laboratory, Roskilde, Denmark (1999). 